\title{
Comparison between histochemical and immunohistochemical methods for diagnosis of sporotrichosis
}

\author{
M E A Marques, K I R Coelho, M N Sotto, C E Bacchi
}

\begin{abstract}
Aims: To compare the efficacy of histochemical and immunohistochemical methods in detecting forms of Sporothrix schenckii in tissue.

Methods: Thirty five cutaneous biopsy specimens from 27 patients with sporotrichosis were stained by histochemical haematoxylin and eosin, periodic acid Schiff, and Gomori's methenamine silver methods and an immunohistochemical (avidin-biotin complex immunoperoxidase) (ABC) technique associated with a newly produced rabbit polyclonal antibody anti-Sporothrix schenckii.

Results: A total of $29(83 \%)$ cases were positive by the ABC method used in association with anti-Sporothrix schenckii rabbit polyclonal antibodies. Histochemical methods, using silver staining, periodic acid Schiff, and conventional haematoxylin and eosin detected $37 \%$, $23 \%$, and $23 \%$ of forms of $S$ schenckii, respectively. The ABC technique was significantly more reliable than periodic acid Schiff and silver staining techniques.

Conclusions: It is concluded that immunostaining is an easy and rapid method which can efficiently increase the accuracy of the diagnosis of sporotrichosis in human tissue.
\end{abstract}

(F Clin Pathol 1992;45:1089-1093)

Sporotrichosis is a subacute or chronic deep mycosis of the skin and regional lymphatics that results from the percutaneous introduction of Sporothrix schenckii. This dimorphic fungus is found throughout the world, and in mammalian tissues is yeast-like, appearing as spherical or cigar-shaped budding cells. Sporotrichosis may be suspected in the presence of a positive skin test using sporotrichin, but should be confirmed by culture or biopsy of the lesion with identification of $S$ schenckii. However, it is not always possible to recognise differing forms of the organism in tissue sections. This seems to be especially true in cases of sporotrichosis reported from the United States ${ }^{1}$ and Europe. ${ }^{2}$ Therefore, the development of an easy, rapid, reliable and specific assay for the identification of $S$ schenckii and subsequent confirmation of the diagnosis of sporotrichosis in biopsy specimens would be very useful.

\section{Methods}

A total of 35 cutaneous biopsy specimens from 27 patients with sporotrichosis confirmed by culture were studied. Clinical data were obtained from the medical records of each patient.

All skin biopsy specimens were fixed in $10 \%$ formalin solution and paraffin wax embedded. Five slides of $5 \mu \mathrm{m}$ thick multiple serial sections were prepared from each block. Three sequential slides from each case were stained by haematoxylin and eosin, periodic acid Schiff, and Gomori's methenamine silver, respectively. Those histological sections were analysed with special attention to morphological findings and screened for the presence of $S$ schenckii. The remaining two slides were used for immunohistochemical staining.

\section{ANTIGEN PREPARATION}

A pool derived from four different cultures of $S$ schenckii was extracted by washing whole cells of $S$ schenckii three times in phosphate buffered saline, followed by ultracentrifugation (30 cycles of two minutes each at 60-70 cycles per second for 20 minutes). The resulting supernatant fluid was put through a Millipore filter, yielding a final protein concentration of $3.0 \mathrm{mg} / \mathrm{ml}$.

\section{ANTIBODY PRODUCTION}

A rabbit was immunised subcutaneously (3 doses) and intravenously (5 doses). The subcutaneous inoculum containing $1 \mathrm{ml}$ of the $S$ schenckii antigen preparation and $1 \mathrm{ml}$ of Freund's complement adjuvant was given at two sites on the back of the animal. The intravenous inocula containing $0.5 \mathrm{ml}$ of the $S$ schenckii antigen was injected in each of the marginal veins of the ear. The first seven inocula were given at variable intervals of two to 10 days with the last inoculum (8th) given subcutaneously 30 days after the 7 th. When the titre in the serum reached $1 / 32$, the rabbit was bled and the total serum separated and designated Total Anti-S schenckii (TASS) antiserum. Part of the serum was used for the purification of the IgG class by Sepharose gel chromatography ${ }^{3}$ and named Anti-S schenckii antibody, class IgG (ASSIgG). To diminish crossreactivity with other fungi, the TASS and ASSIgG were also absorbed with a pool of yeast cultures samples of strains of the fungus Paracoccidiodes brasilienses according to a previously described technique. ${ }^{4}$

IMMUNOHISTOCHEMICAL STAINING

Two slides from each case containing serial sections of tissue were stained by the avidinbiotin and immunoperoxidase method (ABC) using 3,3'-diaminobenzidine as the chromogen, as described previously. ${ }^{5}$ TASS and 
ASSIgG were used as primary antibodies at 1 in 500 and 1 in 100 dilutions, respectively, based on the use of positive and negative controls. Sections were counterstained with

Table 1 Results of histochemical and immunohistochemical methods for detection of forms of Sporothrix schenckii in cutaneous biopsy specimens

\begin{tabular}{|c|c|c|c|c|c|}
\hline \multirow[b]{2}{*}{ Case Nos } & \multicolumn{3}{|c|}{ Histochemistry } & \multicolumn{2}{|c|}{ Immunohistochemistry } \\
\hline & $H E$ & PAS & $G M S$ & TASS & $A S S I g G$ \\
\hline 1 & + & - & - & + & + \\
\hline 2 & + & + & - & + & + \\
\hline 3 & - & + & - & + & - \\
\hline 4 & - & - & - & + & - \\
\hline 5 & - & - & - & + & + \\
\hline 6 & - & - & - & + & + \\
\hline 7 & - & - & - & + & + \\
\hline 8 & - & - & - & + & - \\
\hline 9 & - & - & - & - & - \\
\hline 10 & - & - & - & - & + \\
\hline 11 & - & - & + & - & + \\
\hline 12 & - & - & - & - & + \\
\hline 13 & - & - & + & - & - \\
\hline 14 & - & - & + & - & + \\
\hline 15 & - & + & + & - & - \\
\hline 16 & - & - & + & - & - \\
\hline 17 & + & - & + & - & - \\
\hline 18 & + & + & - & + & + \\
\hline 19 & + & - & + & + & - \\
\hline 20 & + & + & + & + & + \\
\hline 21 & - & + & - & + & + \\
\hline 22 & + & + & - & - & + \\
\hline 23 & + & - & + & + & + \\
\hline 24 & - & - & - & - & - \\
\hline 25 & - & + & + & + & + \\
\hline 26 & - & - & + & + & + \\
\hline 27 & - & - & - & + & + \\
\hline 28 & - & - & - & + & + \\
\hline 29 & - & - & - & - & + \\
\hline 30 & - & - & + & + & + \\
\hline 31 & - & - & - & - & + \\
\hline 32 & - & - & + & + & + \\
\hline 33 & - & - & - & - & - \\
\hline 34 & - & - & - & + & + \\
\hline 35 & - & - & - & - & - \\
\hline Total positive & $8(23 \%)$ & $8(23 \%)$ & $13(37 \%)$ & $20(57 \%)$ & $23(66 \%)$ \\
\hline
\end{tabular}

HE: haematoxylin and eosin

PAS: periodic acid Schiff

GMS: Gomori's methenamine silver

Table 2 Results of immunostaining in skin biopsy specimens for detection of forms of Sporothrix schenckii using TASS and ASSIgG as primary antibodies

\begin{tabular}{|c|c|c|c|c|}
\hline \multirow[b]{3}{*}{ Case Nos } & \multicolumn{4}{|c|}{ Immunostaining ( $A B C$ method) } \\
\hline & \multicolumn{2}{|l|}{ TASS } & \multicolumn{2}{|l|}{$A S S I g G$} \\
\hline & Fungi & Antigen & Fungi & Antigen \\
\hline 1 & + & + & + & + \\
\hline 2 & + & + & + & + \\
\hline 3 & + & - & - & - \\
\hline 4 & + & - & - & - \\
\hline 5 & + & + & + & + \\
\hline 6 & + & + & + & + \\
\hline 7 & + & + & + & + \\
\hline 8 & + & - & - & - \\
\hline 9 & - & - & - & - \\
\hline 10 & - & - & + & - \\
\hline 11 & - & - & + & - \\
\hline 12 & - & + & + & + \\
\hline 13 & - & + & - & - \\
\hline 14 & - & - & + & - \\
\hline 15 & - & - & - & - \\
\hline 16 & - & - & - & - \\
\hline 17 & - & - & - & - \\
\hline 18 & + & + & + & + \\
\hline 19 & + & - & - & - \\
\hline 20 & + & + & + & + \\
\hline 21 & + & - & + & - \\
\hline 22 & - & - & + & + \\
\hline 23 & + & + & + & + \\
\hline 24 & - & - & - & - \\
\hline 25 & + & + & + & + \\
\hline 26 & + & - & + & - \\
\hline 27 & + & - & + & - \\
\hline 28 & + & - & + & - \\
\hline 29 & - & - & + & + \\
\hline 30 & + & + & + & + \\
\hline 31 & - & - & + & + \\
\hline 32 & + & - & + & - \\
\hline 33 & - & + & - & - \\
\hline 34 & + & + & + & - \\
\hline 35 & - & - & - & - \\
\hline Total positive & $20(57 \%)$ & $14(40 \%)$ & $23(66 \%)$ & $14(40 \%)$ \\
\hline
\end{tabular}

haematoxylin. To evaluate antibody specificity, histological sections of mouse testis, which had been experimentally infected with $S$ schenckii and rich in fungi, were used as positive controls. Non-immunised rabbit serum as primary antibody and tissue rich in Paracoccidioides brasilienses were used as negative controls. Endogenous peroxidase was blocked by incubating the tissue in a $0.5 \%$ solution of $\mathrm{H}_{2} \mathrm{O}_{2}$ in absolute methanol for 30 minutes. All four cases of skin lesions of paracoccidioidomycosis stained with both TASS and ASSIgG antibodies did not react.

The immunostaining using TASS and ASSIgG antibodies adsorbed with $S$ schenckii cultures was negative in positive control tissue (mouse testis) rich in $S$ schenckii.

\section{Results}

CLINICAL AND MORPHOLOGICAL FINDINGS

The 27 patients were divided according to clinical presentation as follows: $13(48 \%)$ cutaneous-lymphatic form; 12 (44\%) local, isolated cutaneous form; and two ( $8 \%$ ) disseminated cutaneous form. Morphologically, epithelial hyperplasia was observed in $80 \%$ of the cases, intraepidermic microabscesses in $71 \%$, and an epithelioid granulomatous process, generally with central purulence, in $91 \%$. The inflammatory infiltrate surrounding the granulomas was in most cases of the lymphoplasmocytic type.

\section{HISTOCHEMICAL AND IMMUNOHISTOCHEMISTRY} FINDINGS

Results of the detection of $S$ schenckii in tissues using histochemical methods are summarised in table 1. Sporothrix schenckii was identified in $13(37 \%)$ cases, eight (23\%) cases and eight (23\%) cases using, respectively, Gomori's methenamine silver, periodic acid Schiff, and haematoxylin and eosin, staining. On periodic acid Schiff staining the fungi were more characteristic, having a more intensely stained wall and lighter protoplasm (fig 1A). Positive staining by periodic acid Schiff of the cytoplasm of giant cells and macrophages in the granulomas was also noted (fig 1B). The forms of $S$ schenckii identified by histochemical staining, specially Gomori's methenamine silver, were rare, round or oval, generally small yeastlike structures, with occasional single buds (fig 1C).

Table 1 also summarises the immunohistochemical findings for the detection of $S$ schenckii. Using ABC immunostaining, $S$ schenckii was detected in $20(57 \%)$ cases using the TASS antibody and in $23(66 \%)$ cases with the ASSIgG antibody. Using TASS or ASSIgG antibodies, or both, yeast-like forms and putative antigens of $S$ schenckii were visualised in 29 out of $35(83 \%)$ cases. (fig 1D) (table 2). The putative antigens of $S$ schenckii were characterised by a granular, uniform type of staining in the cytoplasm of the giant cells as well as in macrophages within the granulomas (figs $1 \mathrm{E}$ and $1 F$ ). This observation was similar with both primary antibodies (TASS and ASSIgG). The detection of putative antigens of $S$ 

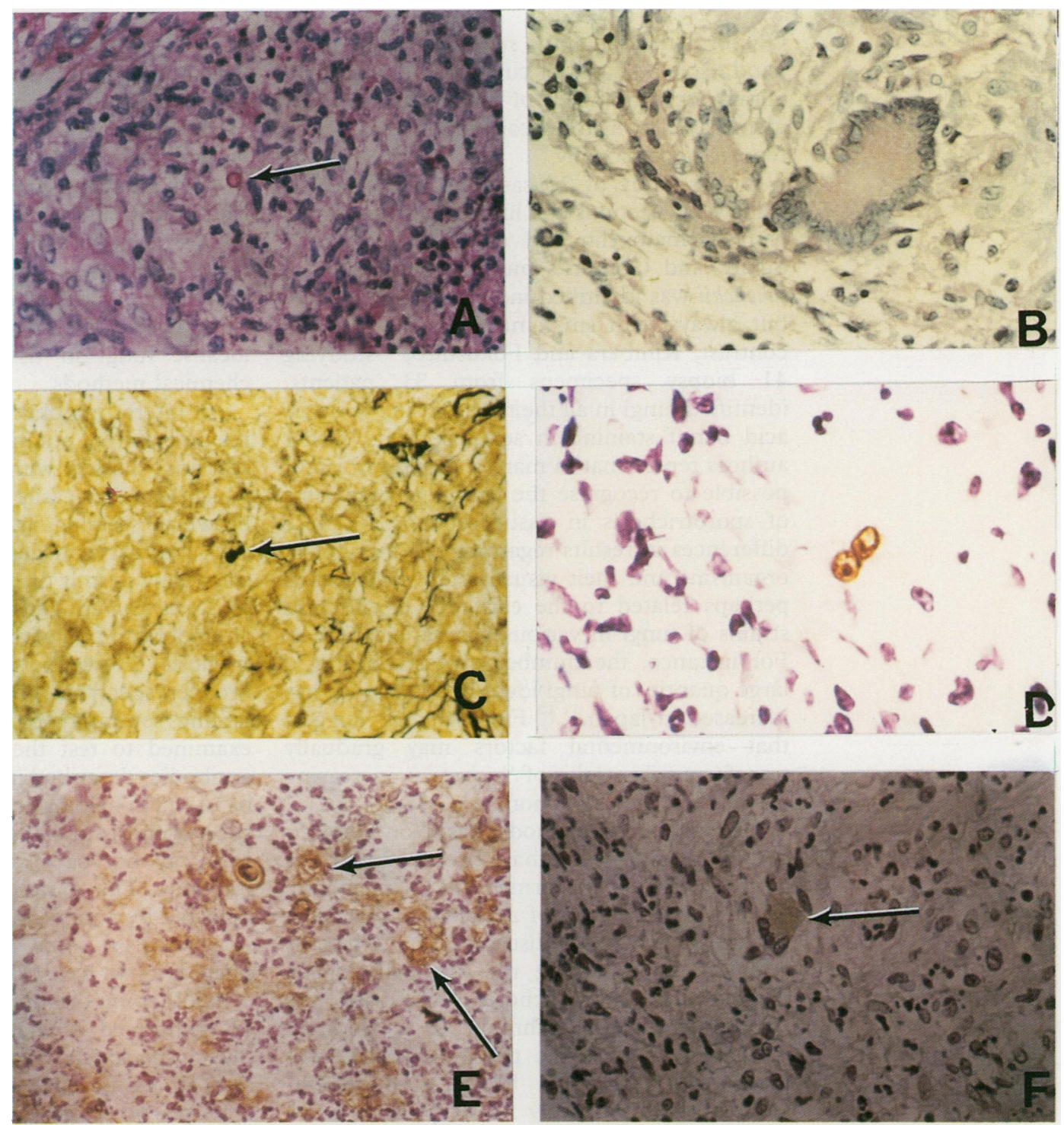

Figure 1 A Cutaneous tissue stained by periodic acid Schiff showing one round form of $S$ schenckii in the centre of suppuration (arrow).

$B$ Cutaneous tissue with periodic acid Schiff positive multinucleated giant cells in the centre of a granuloma.

$C$ Cutaneous tissue stained by Gomori's methenamine silver, showing one small yeast-like form of $S$ schenckii (arrow).

$D$ Cutaneous tissue. Yeast-like forms of $S$ schenckii stained by anti-S schenckii rabbit antibody (avidin-biotin immunoperoxidase preparation).

$E$ Cutaneous tissue. Yeast-like forms and putative antigens (arrows) of $S$ schenckii stained by anti-S schenckii rabbit antibody (avidin-biotin immunoperoxidase preparation).

$F$ Cutaneous tissue. Multinucleated giant cell showing positivity in the cytoplasm (arrow) for anti-S schenckii rabbit antibody (avidin-biotin immunoperoxidase preparation).

schenckii occurred in $14(40 \%)$ of the cases using either antibody (TASS or ASSIgG) (table 2).

Table 3 compares the results of the histochemical and immunohistochemical techniques. The $A B C$ method was the most

Table 3 Comparison of histochemical and immunohistochemical methods for detection of forms of Sporothrix schenckii in tissues

\begin{tabular}{lll}
\hline Methods & Positive cases/Total & $\begin{array}{l}\text { Percentage of } \\
\text { positive cases }\end{array}$ \\
\hline ABC & $27+/ 35$ & 77 \\
HE & $8 / 35$ & 23 \\
PAS & $8+135$ & 23 \\
GMS & $13+/ 35$ & 37 \\
PAS + HE + GMS & $19+/ 35$ & 51 \\
ABC + PAS + HE + GMS & $31+/ 35$ & 89 \\
\hline
\end{tabular}

ABC: Avidin-biotin immunoperoxidase method. HE: haematoxylin and eosin. PAS: periodic acid Schiff; GMS: Gomori's methenamine silver. sensitive in detecting $S$ schenckii $(77 \%)$, followed by Gomori's methenamine silver (37\%) and periodic acid Schiff or haematoxylin and eosin, each with $23 \%$ positivity. Considering the three histochemical methods together, $S$ schenckii was detected in $19(54 \%)$ cases.

There was no difference among the various clinical forms of sporotrichosis studied in relation to the histopathological findings and the quantity of fungi encountered in the lesions.

\section{Discussion}

Histopathological examination is not the best diagnostic method for the diagnosis of sporotrichosis, because $S$ schenckii may be difficult to identify in histological sections stained with routine histochemical staining such as haematoxylin and eosin, periodic acid Schiff and 
Gomori's methenamine silver. ${ }^{67}$ On the other hand, isolated cases of cutaneous lesions containing large numbers of fungi that are easily identifiable in histological sections have been reported occasionally. ${ }^{89}$

In our study of 27 cases of sporotrichosis using serial sections and histochemical staining with haematoxylin and eosin, periodic acid Schiff, and Gomori's methenamine silver, $S$ schenckii was identified in about half the cases but always with small numbers of fungi. In contrast, Kimbara and Fukushiro, ${ }^{10}$ studying 41 biopsy specimens from 31 patients, identified fungi in all their cases using periodic acid Schiff staining in serial sections. Other authors report that in many instances, it is not possible to recognise the causative organisms of sporotrichosis in tissue sections. ${ }^{12}$ These differences in results regarding the number of organisms and their visualisation in tissues is perhaps related to the existence of different strains of fungi in various geographical areas. For instance, the number of cases having a large quantity of fungi identified in tissues has increased in Japan. ${ }^{112}$ Findlay et $a l^{13}$ suggest that environmental factors may gradually transform wild strains of $S$ schenckii, increasing the possibility of infection.

Immunological methods, based on the antigen-antibody reaction, have been developed as a rapid and easily performed specific technique for the identification of aetiological agents in several infectious diseases. ${ }^{14-16}$ Previous attempts at improving the histopathological diagnosis of sporotrichosis have used the immunoperoxidase technique as an indirect method as well as the peroxidase-antiperoxidase technique. ${ }^{141517}$ Uribe et al demonstrated $100 \%$ positively for $S$ schenckii in 18 cases of cutaneous sporotrichosis confirmed by culture using the indirect immunoperoxidase technique, with a specific polyclonal antibody obtained from pooled human sera of patients with sporotrichosis. ${ }^{17}$ Although this result seems promising, the use of human antibody as a primary antibody for immunohistochemistry is not desirable as non-specific background can develop because anti-human immunoglobulin has to be used as a secondary antibody.

Our study of 27 cases stained by the $A B C$ technique, using rabbit polyclonal primary antibodies against $S$ schenckii, demonstrated fungi or the putative antigens of $S$ schenckii in $83 \%$ of the cases. This result is better than any obtained using histochemical methods for the detection of $S$ schenckii: the best histochemical method was silver staining with positive results in $37 \%$ of the cases. In fact, the ABC technique was more sensitive even when all three histochemical methods were combined. These results show the possibility of improving the efficacy of histological diagnosis of this mycosis in cutaneous biopsy specimens using immunohistochemical techniques. It should be emphasised, however, that even with the use of more sensitive staining techniques such as the ABC method, multiple serial sections of the same tissue ought to be examined and the slides screened very carefully because the fungi can be rare and are generally small and isolated. Another advantage of using immunohistochemical techniques is better visualisation of the fungi which, on the slides, stain dark brown, in contrast to the light blue background of haematoxylin counterstaining. By comparison Gomori's methenamine silver staining gives a black positivity and it is not specific for fungus because it stains other structures in tissues such as reticular fibres. These characteristics of silver staining make it more difficult to localise fungi in tissue than immunohistochemical methods.

An interesting observation in our study was the detection of putative $S$ schenckii antigens in sections stained with ABC. This positivity, which can be easily identified as granular, brownish staining in the cytoplasm of giant cells and macrophages inside granulomas, may eventually permit the study of the distribution of $S$ schenckii antigens and could contribute to an understanding of the pathogenesis of sporotrichosis. However, further studies with a broader spectrum of biopsy specimens containing several types of fungi has yet to be examined to test the total specificity of the antibodies described in this study. It might also be useful in helping to confirm the diagnosis of sporotrichosis in cases where the entire organisms can not be visualised by histochemical methods. This putative antigen can also be detected by periodic acid Schiff staining in the cytoplasm of some giant cells and macrophages, as we have noted in our study.

Another important observation that ought to be mentioned is related to the depth of the biopsy specimens. Superficial ones may not be satisfactory because they represent only the hyperplastic epidermis and a small quantity of dermis. On the other hand, deeper specimens are more likely to contain larger numbers of fungi present in the dermis and even in the subcutaneous tissue. The material obtained with small punches may not be sufficient to make serial sections for the identification of the agent, especially when multiple staining techniques need to be performed.

We therefore conclude that the ABC immunoperoxidase technique using specific antibodies against $S$ schenckii is more sensitive and specific than the histochemical methods for the detection of fungi in histological sections of biopsy specimens infected with sporotrichosis. However, we should emphasise that our comparative analysis of histochemical and immunohistochemical results for $S$ schenckii detection shows that the combination of two techniques augments the likelihood of making the diagnosis of sporotrichosis in cutaneous biopsy specimens. The immunoperoxidase technique also offers the advantage of using specific antibodies, thus guaranteeing a more precise diagnosis than those based on the morphological characteristics of the fungi determined with empirical techniques as haematoxylin and eosin, periodic acid Schiff, and Gomori's methenamine silver. ${ }^{18}$

We thank Mara S Angela, Celene $M$ de Souza, and Dalva A Ferracini for performing the histochemical and immunohistochemical studies, Gastão Chamas and Marino A da Cunha for 
the antigen preparations, and Rosangela M P de Camargo and Alberto Salebian for cultures of the fungi.

1 Segal RJ, Jacobs PH. Sprotrichosis. Int $\mathcal{f}$ Dermatol 1979;18:639-44.

2 Male O. Diagnostische und therapeutische Problem bei der kutanen Sporotrichose. Z Hautkr 1974;49:505-15.

3 Ey PL, Prouse SJ, Jenkin CR. Isolation of pure IgG1, IgG2, IgG2b immunoglobulins of mouse serum using protein-A sepharose Immunochemistry 1978;15:429-36.

4 Iabuki K Blastomicose sul Americana experimental do hamster. (Ph.D thesis). Faculdade de Ciências Médicas e Biológicas de Botucatu, Botucatu, Sào Paulo, Brazil: 1973:182pp.

5 Hsu SM, Raine L, Fanger H. Use of avidin-biotin peroxidase complex $(\mathrm{ABC})$ in immunoperoxidase techniques: $A$ comparison between $\mathrm{ABC}$ and unlabelled (PAP) procedures. F Histochem Cytochem 1981;29:577-80.

6 Chandler FW, Kaplan W, Ajello L. Color atlas and test of histopathology of mycotic diseases. Chicago: Year Book Medical Publishers, 1980:281-7.

7 Rippon JW. Sporotrichosis. In: Medical mycology 3rd ed. Philadelphia, WB Saunders, 1988:325-52.

8 Hiruma M, Kagawa S. A case of sporotrichosis with numerous fungal elements phagocytized by polymorphonuclear leucocytes. Mykosen 1986;29:59-63.
9 Geyer GR, Severo LC. Formas teciduais dos Sporothrix Schenckii em lesão humana. Apresentaçào de caso. Rev Bras Patol Clin 1983;19:70-1.

10 Kimbara T, Fukushiro R. Fungal elements in tissues of sporotrichosis. Mykosen 1982;26:35-41.

11 Hachisuka H, Sasai Y. A peculiar case of sporotrichosis. Dermatologica 1980;160:37-40

12 Yamano T, Mazaki H, Honbo S, Urabe H. Sporotrichosisthe study on cases with numerous fungal elements. $\mathcal{I p s ~}^{\mathcal{F}}$ Med Mycol 1985;26:50.

13 Findlay GH, Wismer HF, Dreyer L. Studies on sporotrichosis. Mycopathologia 1986;87:85-93.

14 Russel B, Beckett JH, Jacobs PH. Immunoperoxidase localization of Sporothrix schenckii and Cryptococcus neoformans. Arch Dermatol 1979;115:433-5.

15 Moskowitz LB, Ganjei P, Weissman JZ, Cleary JT, Penneys SN, Nardji M. Immunohistologic identification of fung in systemic and cutaneous mycosis. Arch Pathol Lab Med 1986;110:433-6.

16 Kobayashi $K$, Hayama $M$, Hotchi $M$. The application of immunoperoxidase staining for detection of causative fungi in tissue specimens of mycosis I. Mycopathologia 1988;102:107-13.

17 Uribe F, Zuluaga A, Leon W, Restrepo A. Aspectos histológicos de la esporotricosis y busqueda del agente etiológico por métodos, immunoenzimáticos. Acta Médica Colombiana 1985;10:65-74.

18 Schwartz JN. Specific fungal diagnosis. Arch Pathol 1986; 110:369-70. 